\title{
NAMEN DER INFEKTIONSKRANKHEITEN IM DEUTSCHEN UND NIEDERLÄNDISCHEN
}

\author{
Ewa Maria Majewska \\ Uniwersytet Warszawski, Poland
}

\begin{abstract}
Names of infectious diseases in German and Dutch
Abstract: This article refers to names of infectious diseases, which are constructed in many different ways in German and Dutch. The research corpus was collected from German and Dutch specialized medical lexicons. The names of infectious diseases were introduced and examined from the point of view of semantic and morphological analysis. The names examined were divided into groups and compared with each other. German and Dutch are two West Germanic languages which show many morphological similarities. However, the contrastive study of the names of infectious diseases in both languages allows us to see several differences between them as well. The immense influence of classical languages such as Greek and Latin and the existence of new medical terms in English are some of the main features of this nosological vocabulary.
\end{abstract}

Keywords: infectious diseases, medical vocabulary, languages for special purposes, medical language.

\section{Einleitung}

Krankheiten stehen in einer Beziehung zum Sacrum und Profanum. In der Bibel finden wir verschiedene Beispiele der Infektionskrankheiten, die als Strafe Gottes angesehen wurden. Als eine der bekanntesten Krankheiten aus dem Altertum gilt Lepra. Im Neuen Testament finden wir beim Evangelisten Markus die Stelle über die Genesung des Aussätzigen von Jesus (Mk 1, 40-45). Ein anderes Beispiel, wo das Fieber auftritt, das hier auch als Krankheit beschrieben wurde, betrifft die Geschichte, in der Jesus die am Fieber erkrankte Schwiegermutter des Heiligen Petrus geheilt hat. (Mk 1, 29-31). Im Alten Testament kam die Pest vor, die als Gericht Gottes über Sein rebellisches Volk erwähnt wurde (2 Sam 24,15-16).

Vom linguistischem Standpunkt aus könnte man darüber streiten, ob man Namen der Krankheiten als Eigennamen oder eigentlich als Gattungsnamen ansehen sollte. Solchen Komposita wie Blutvergiftung oder Lungenentzündung sollte man ohne weiteres als Gattungsnamen betrachten. Dagegen werden solche Krankheitsbezeichnungen wie Ruhr, Mumps oder Scharlach eher für Eigennamen gehalten, vor allem wenn sie ohne Artikel und nur im Singular verwendet werden (Bauer 1996: 1619). Probleme mit der richtigen Einordnung können Krankheitsnamen mit Eponymen liefern, die von den 
Personennamen gebildet worden sind. Unter diesem Aspekt sind Krankheitsnamen als eine Art Übergangsform zwischen den Eigennamen und Gattungsnamen anzusehen (Bauer, ebenda).

Für Krankheitsbezeichnungen gibt es keine international vereinbarte Nomenklatur im Gegensatz zur Anatomie. Jedoch ist eine fachliche Verständigung möglich, obwohl für manche Krankheiten mehrere oder viele Namen bestehen (Schulze 2003: 23). Als Beispiel kann die Ahornsirupkrankheit genannt werden, die auch unter dem Namen Leuzinose oder Verzweigtkettenkrankheit (aus dem engl. Maple syrup urine disease) bekannt ist. Zahlreiche Krankheiten kommen mit mindestens einem Synonym vor, z.B. Diphtherie (echter Krupp), Keuchhusten (Pertussis) oder sogar mit zwei Synonymen, z.B. Mumps (Ziegenpeter oder Parotitis epidermica).

Das Alter der Krankheitsnamen ist unterschiedlich, je nachdem, wann die betreffende Erkrankung erstmalig beschrieben wurde. So ist die Bezeichnung Karzinom schon in den Schriften des Corpus Hippocraticum zu finden, also etwa 2500 Jahre alt. Dagegen ist der Name Appendizitis (Wurmfortsatzentzündung nicht älter als 150 Jahre (Schulze 2003: 24).

\section{Definition und Korpus}

Infektionskrankheit ist nach DUDEN Wörterbuch Medizinischer Fachbegriffe (2007: 393): „durch Eindringen und Vermehrung infektionstüchtiger (virulenter) pathogener Mikroben und durch Einwirkung giftiger Stoffwechselprodukte (Toxine) dieser Erreger hervorgerufene Krankheit mit meist typischem Verlauf".

Das gesammelte Korpus umfasst Namen von Infektionskrankheiten, die in der deutschen und niederländischen Fachsprache verwendet werden. Es wurde eine Liste von Infektionskrankheiten aufgestellt, die im deutschsprachigen Lexikon der Krankheiten und Untersuchungen genannt und beschrieben worden sind. Diese Liste enthält 49 Krankheiten, für die manchmal mehrere Synonyme im Deutschen vorliegen: insgesamt 66 Bezeichnungen:

AIDS, Aspergillose, Borreliose, Botulismus, Cholera (Syn. Gallenbrechdurchfall), CreutzfeldtJakob-Erkrankung, Denguefieber (Syn. Siebentagefieber), Echinokokkose, Enzephalitis, Gasbrand, Gelbfieber (Syn. Ochropyra), Hepatitis, Herpes simplex, Herpes zoster (Syn. Gürtelrose), Impetigo contagiosa (Syn. Grindflechte, Borkenflechte), Katzenkratzkrankheit, Lassa-Fieber, Legionellose, Lepra, Listeriose, Malaria, Marburg-Viruskrankheit, Masern, Meningitis, Milzbrand, Mumps (Syn. Ziegenpeter, Parotitis), Neugeborenenherpes, Papageienkrankheit (Syn. Psittakose), Peritonitis, Pertussis (Syn. Keuchhusten), Pest, Pfeiffer'sches Drüsenfieber, Pneumonie, Pocken, Ringelröteln (Syn. Erythema infectiosum, Erythema variegatum, Fünfte Krankheit), Röteln, Ruhr, SARS, Scharlach, Skabies, Syphilis, Tetanus, Tollwut (Syn. Rabies, Lyssa), Toxoplasmose, Trypanosomiasis, Tuberkulose, Vogelgrippe, Windpocken, Wundrose (Syn. Erysipel).

Die Beispielsätze, die die Krankheitsnamen enthalten, stammen teilweise auch vom Duden Wörterbuch medizinischer Fachbegriffe (2007). 
Im Niederländischen sind im Falle von denselben 49 Krankheiten die folgenden 86 Bezeichnungen gebräuchlich:

Aspergillose, Aids, borreliose (Syn. Lyme-borreliose), bof (Syn. parotitis epidermica, epidemische parotitis), botulisme, cholera, dengue (Syn. knokkelkoorts, vijfdaagse koorts, dandy fever, forest fever, rangoonkoorts, Van der Scheerse koorts), dysenterie/dysenteria, echinokokkose, Erythema infectiosum (Syn. megalerythema epidermicum, exantema variabile, vijfde ziekte), encefalitis, gasgangreen, gele koorts (Syn. ochropyra), hepatitis (Syn. leverontsteking), herpes neonatorum, herpes simplex (Syn. herpes febrilis), herpes zoster (Syn. gordelroos, zona), impetigo contagiosa (Syn. impetigo vulgaris), kattenkrabziekte, lassa-koorts/ lassakoorts (Syn. hemorragische Lassa-koorts), legionellose, lepra (Syn. melaatsheid, ziekte van Hansen, morbus Hansen), listeriosis, malaria, marburgvirus, mazelen, meningitis, miltvuur (Syn. anthrax/antrax, carbunculus contagiosus, Siberische pest), papegaaienziekte (Syn. psittacose/psittacosis), peritonitis, pertussis (Syn. kinkhoest), pest, pokken, rabiës (Syn. hondsdolheid, lyssa), rodehond, roodvonk (Syn. scarlatina), SARS, scabies (Syn. schurft), syfilis, tetanus, tuberculose/tuberculosis, toxoplasmose/toxoplasmosis, trypanosomiasis (Syn.slaapziekte, safariziekte), vogelgriep, waterpokken (Syn. varicella), wondroos (Syn. belroos, erysipelas), ziekte van Creutzfeldt-Jakob (Syn. prionziekte), ziekte van Pfeiffer (Syn. mononucleose, mononucleosis infectiosa, klierkoorts van Pfeiffer).

Die Namen der Krankheiten im niederländischen Teil der Untersuchung sind Äquivalente der deutschen Bezeichnungen. Die Beispiele stammen aus dem niederländischsprachigen Wörterbuch des medizinischen Fachwortschatzes Pinkhof Geneeskundig Woordenboek von Van Everdingen (2006).

Für manche Krankheiten gibt es mehr als eine Bezeichnung und die Zahl der Namenvarianten ist nicht immer in beiden Sprachen gleich, obwohl das Deutsche und das Niederländische, als zwei Westgermanische Sprachen, einander ähneln.

\section{Morphologische Einteilung der Namen von Infektionskrankheiten im Deutschen}

Unter den Krankheitsnamen finden sich verschiedene Wortbildungstypen. Es gibt Bezeichnungen, die nur im Plural vorkommen, sog. Pluralia tantum:

Pluralia tantum

- „Röteln sind eine akute Infektion, hervorgerufen durch das Rötelnvirus und verursachen Fieber, Lymphknotenschwellung, Hautausschlag und weitere mögliche Krankheitserscheinungen"(LKU, 930).

- „Die Masern heilen meist folgenlos aus und führen zu einer lebenslangen Immunität" (LKU, 629).

- „Die Pocken wurden durch umfangreiche Impfung, Quarantäne- und Beobachtungsmaßnahmen ausgerottet" (LKU, 847).

Pendents: $d$. Röteln $\rightarrow n l$. rodehond (PGW, 999), $d$. Masern $\rightarrow n l$. mazelen $(\mathrm{PGW}, 719), d$. Pocken $\rightarrow n l$. pokken (PGW, 1226), $d$. Windpocken $\rightarrow n l$. waterpokken (PGW,1224) 


\section{Determinativkomposita}

Unter den Namen der Infektionskrankheiten im Deutschen finden sich zahlreiche Beispiele für Determinativkomposita vom Typ: Substantiv + Substantiv und Adjektiv + Substantiv:

\section{Typ A: Substantiv + Substantiv}

- „Als Milzbrand werden Infektionen mit dem aeroben Sporenbildner Bacillus anthracis bezeichnet“. (LKU, 651).

- „Als Gasbrand werden schwere Wundinfektionen, die typischerweise von Gasbildung begleitet sind, bezeichnet" (LKU, 336).

- „Das Ringelröteln -ist die Manifestation einer Infektion durch das Parovirus B19“ (LKW, 297).

- „Windpocken sind eine akute Erkrankung mit juckendem Hautausschlag, die durch das Varicella-zoster-Virus hervorgerufen wird“ (LKU, 1352).

- „Die Wundrose (gr. Erysipel) - ist eine Infektion mit $\beta$-hämolysierenden Streptokokken der Gruppe A“ (LKU, 296).

Pendents: $d$. Milzbrand $\rightarrow$ nl.miltvuur (Syn. anthrax/antrax (G), carbunculus contagiosus, Siberische pest) (PGW,70); d. Gasbrand $\rightarrow n l$. gasgangreen (PGW, 439), d. Ringelröteln (Syn. Erythema infectiosum, Erythema variegatum, Exanthema infectiosum, Fünfte Krankheit) $\rightarrow$ nl. erythema infectiosum (Syn. megalerythema epidermicum, exantema variabile, vijfde ziekte) (PGW, 375), $d$. Windpocken $\rightarrow n l$. waterpokken, varicella (PGW, 1224), $d$. Wundrose (g. Erysipel) $\rightarrow n l$. wondroos (Syn. belroos, erysipelas) (PGW, 374).

\section{Determinativkomposita mit Tiernamen}

- „Katzenkratzkrankheit- seltene Viruserkrankung, die hauptsächlich durch Katzenkratzer oder Katzenbisse auf den Menschen übertragen wird und sich in Fieber, Geschwürbildung und Lymphknotenschwellung äußert" (DUDEN WMF, 422).

- „Vogelgrippe - Viruskrankheit, die durch das Influenzavirus H5N1, bezeichnet nach speziellen Oberflächenproteinen, hervorgerufen wird“ (DUDEN WMF, 821)

- Papageienkrankheit, Syn.Psittakose (Gr.psittakos,Papagei') - durch Chlamydien hervorgerufene, auf den Menschen übertragbare bakterielle Infektionskrankheit“ (DUDEN WMF, 649).

Pendents: $d$. Katzenkratzkrankheit $\rightarrow n$ l. kattenkrabziekte (PGW, 597), $d$. Vogelgrippe $\rightarrow n l$. vogelgriep (PGW, 1260), $d$. Papageienkrankheit $\rightarrow n l$. papegaaienziekte, psittacose/psittacosis (PGW, 928)

\section{Determinativkomposita: Typ B: Adjektiv + Substantiv}

- „Die Tollwut (Syn. Rabies, Lyssa) ist eine akute Erkrankung des Zentralnervensystems, die durch das Tollwutvirus hervorgerufen und meist über Speichel von Tieren übertragen wird " (LKU, 1076).

- „Gelbfieber (auch gr. Ochropyra) - tropische, durch Mücken übertragene Virusinfektionskrankheit, deren Symptome Schüttelfrost, hohes Fieber, Gelbsucht u.a. sind' (DUDEN WMF, 322). 
Pendents: $d$. Tollwut $\rightarrow n l$. rabiës, hondsdolheid, lyssa (PGW,945), d. Gelbfieber $\rightarrow n l$. gele koorts, ochropyra (PGW, 445).

\section{Hybride Bildungen}

- „Denguefieber - (Syn. Siebentagefieber), span. Dengue ,Ziererei“ wegen des eigentümlichen Ganges der Kranken, eine tropische Viruskrankheit' (DUDEN WMF, 226). „Dengue - deutet auf eine schmerzbedingte, auffällig eigenartige Veränderung der Körperhaltung und Verhaltensweise hin.

- „Ein Neugeborenenherpes kann sich auch entwickeln, wenn sich das Kind kurz nach der Geburt durch eine Kontaktperson mit Herpes (z.B. Herpes labialis) infiziert" (LKU, 723).

\section{(gr. Herpes- ,Hautgeschwür $\left.{ }^{c}\right)$}

Pendents: $d$. Denguefieber $\rightarrow n l$. dengue (Syn. knokkelkoorts, vijfdaagse koorts, dandy fever, forest fever, rangoonkoorts, Van der Scheerse koorts) (PGW, 290), $d$. Neugeborenenherpes $\rightarrow n l$. herpes neonatorum (PGW, 506).

\section{Eponym als Glied des Kompositums}

- „Eine Variante der Creutzfeldt-Jakob-Erkrankung gilt als die Folge einer Übertragung von BSE (Bovine Spongiforme Enzephalopathie). BSE -ist eine Prionenerkrankung, die Rinder befällt" (LKU, 212).

Pendents: $d$. Creuzfeldt-Jakob-Erkrankung $\rightarrow n l$. ziekte van Creutzfeldt-Jakob, subacute spongiforme encefalopathie, prionziekte (PGW, 1289).

\section{Geonym als Glied des Kompositums}

- „Marburg-Viruskrankheit - (nach der Stadt Marburg (Lahn), dort erstmals 1967 bei Tierpflegern festgestellt) durch Viren hervorgerufenes hämorrhagisches Fieber mit hoher Letalität" (DUDEN WMF, 485).

- „Lassa-Fieber - mit hohem Fieber, Hautblutungen und Lungenentzündung einhergehende Viruserkrankung, die erstmals in Lassa (Nigeria) beobachtet wurde und unbehandelt zum Tod führt" (DUDEN WMF, 457).

Pendents: $d$. Marburg-Viruskrankheit $\rightarrow n l$. marburgvirus (PGW, 685). $d$. LassaFieber $\rightarrow n l$ l lassa-koorts/lassakoorts; hemorragische Lassa-koorts (PGW, 529).

\section{Suffixderrivate von Fremdwörtern:}

Bestimmte große Krankheitsgruppen werden durch Endungen charakterisiert:

Suffix -ose - steht ganz allgemein für eine Krankheit:

- „Die Infektion mit einer Spezies des Schimmelpilzes Aspergillus (z.B. A. fumigatus) wird als Aspergillose bezeichnet" (LKU, 104).

- „Als Borreliose werden Infektionen mit den Schraubenbakterien Borrelia burgdorferi, B. afzelli und B. garinii bezeichnet" (LKU, 165).

- „Listeriose ist eine Infektion mit Listeria monocytogenes (nach dem Chirurgen Joseph Lister), einem grampositiven Stäbchenbakterium" (LKU, 596).

- „Legionellose ist eine Bakterien-Infektion mit Legionella species“. (LKU, 578).

- „Tuberkulose - durch Tuberkelbakterien hervorgerufene chronische Infektionskrankheit, die alle Organe und Systeme befallen kann" (DUDEN WMF, 786) 
- „Als Toxoplasmose wird die Infektion mit Toxoplasma gondii, einem Einzeller (Protozoen) bezeichnet“" (LKU, 1077).

- „Bei der Echinokokkose besteht eine Infektion mit einem EchinococcusErreger" (LKU, 256).

Pendents: $d$. Aspergillose $\rightarrow n l$. aspergillose (PGW, 94), $d$. Borreliose $\rightarrow n l$. borreliose, Lyme-borreliose (PGW, 150), $d$. Listeriose $\rightarrow n l$ listeriosis (PGW, 656), $d$. Legionellose $\rightarrow n l$. legionellose (PGW, 632), $d$. Tuberkulose $\rightarrow n l$. tuberculose, tuberculosis (PGW, 1194), $d$. Toxoplasmose $\rightarrow n l$. toxoplasmose, toxoplasmosis (PGW, 1173), d. Echinokokkose $\rightarrow$ nl. echinokokkose (PGW, 342).

\section{Suffix-ismus:}

Dieses Suffix dient ganz allgemein der Bezeichnung eines krankhaften Zustandes und speziell der Bezeichnung einer Vergiftung (Schulze 2003:27):

- „Als Botulismus wird eine Vergiftung durch Toxine des Bakteriums Clostridium botulinum bezeichnet" (LKU, 167).

Pendents: -ismus/ -isme: $d$. Botulismus $\rightarrow n l$. botulisme/ worstvergiftiging/ allanthiasis (PGW, 152).

Suffix -itis (bezeichnet die Entzündung) (Schulze 2003: 26):

- „Bei der Enzephalitis ist das Hirngewebe akut entzündet“" (LKU, 283).

- „Mit nur geringen Abweichungen zeigt die akute Meningitis ein relativ einförmiges Krankheitsbild“ (LKU, 641).

- „Die Hepatitis ist eine Leberentzündung, bei der Leberzellen zugrunde gehen“ (LKU, 396).

- „Die Peritonitis ist eine lebensbedrohliche, meist bakterielle Entzündung des serösen Bauchfells (Peritoneum)“(LKU, 811).

Pendents: $d$. Enzephalitis $\rightarrow n l$. encefalitis (PGW, 355), $d$. Meningitis $\rightarrow n l$. meningitis (PGW, 699), $d$. Hepatitis $\rightarrow n l$. hepatitis, leverontsteking (PGW, 500), $d$. Peritonitis $\rightarrow n l$. peritonitis (PGW, 860).

\section{Suffix -iasis}

Dieses Suffix bezeichnet als Endung meist „Befallensein“ mit etwas, z.B. mit Steinen, Parasiten oder Giftstoffen (Schulze, 2003: 26):

- „Trypanosomiasis - Syn. Schlafkrankheit, durch Trypanosomen hervorgerufene Infektionskrankheit, die vor allem durch Schlafsucht, nervöse Störungen und Auszehrung gekennzeichnet ist" (DUDEN WMF, 784).

Pendents: $d$. Trypanosomiasis $\rightarrow n l$. trypanosomiasis (Syn. slaapziekte, safariziekte) (PGW, 1193).

\section{Suffix -ie}

- „Als Pneumonie (gr. pneumonos Lunge; pneumonia - Lungenkrankheit) wird eine tiefe Infektion der Lungen bezeichnet" (LKU, 843). $885)$.

Pendents: $d$. Pneumonie $\rightarrow n l$. pneumonie, pneumonia, longontsteking (PGW,

\section{Ableitung vom Fremdwort ohne Suffix:}

- Pest (lat. Pestis = ansteckende Krankheit, Seuche) „ eine Infektionskrankheit, 
die als Beulenpest, Hirnhautentzündung oder Lungenentzündung auftritt" (Schulze 2003: 20).

Pendents: $d$. Pest $\rightarrow n l$. pest (PGW, 865).

\section{Lehnübersetzungen von Fremdwörtern}

- „Scharlach (mlat. Scarlatum = rote Farbe) - akute, durch Streptokokken hervorgerufene Infektionskrankheit hauptsächlich der Kinder, charakterisiert durch Rachenrötung, Angina, kleinfleckiges Exanthem mit Abschuppung ..." (DUDEN WMF, 699).

Pendents: $d$. Scharlach $\rightarrow n l$. roodvonk, scarlatina (PGW, 997).

Ableitungen vom Verb:

- „Ruhr - (mhd. ruore, ahd. hruora, urspr. ,heftige Bewegung; Unruhe (im Unterleib): fiebrige Infektionskrankheit mit Entzündung des Dickdarms und dadurch bedingtem starkem, schleimig-blutigem Durchfall“ (DUDEN DUW, 1275)

Pendents: $d$. Ruhr $\rightarrow n l$. dysenterie, dysenteria (VAN DALE, 741).

Wortgruppe (Adjektiv + Substantiv)

Viele Krankheiten und Syndrome wurden nach ihren Entdeckern oder Erstbeschreibern benannt. Das Eponym in der Form eines Familiennamens steht nach dem lateinischen Wort Morbus „Krankheit': Wenn das Eponym mit dem Namen eines Organs verbunden ist, dann kann dieses oft auf einen pathologischen Zustand dieses Organs hinweisen. Der Familienname des Erstbeschreibers dieser Erkrankung verbirgt sich hinter diesem Eponym. Der Personenname kommt als abgeleitetes Adjektiv in der Funktion eines Attributs vor:

- „Das Pfeiffer'sche Drüsenfieber (Mononukleose, Kusskrankheit) ist eine fieberhafte Erkrankung hauptsächlich jüngerer Menschen, die durch das Epstein-Barr-Virus hervorgerufen und über Speichel übertragen wird“ (LKU, 818).

Pendents: $d$. Pfeiffer'sches Drüsenfieber $\rightarrow n l$. ziekte van Pfeiffer, mononucleose, mononucleosis infectiosa, klierkoorts van Pfeiffer (PGW, 717).

Fremdwörter als Wortgruppe (Substantiv mit adjektivischen Attribut)

- „Herpes simplex - akute Viruserkrankung mit Ausbildung zahlreicher, sich herpetisch zusammenschließender seröser Hautbläschen im Bereich der Schleimhaut der Lippen, der Nase und der äßeren Geschlechtsteile" (DUDEN WMF, 358).

- „Das Herpes zoster (Syn. Gürtelrose) ist eine Erkrankung der Haut aufgrund einer Reaktivierung des Varicella-Zoster-Virus, dem Erreger der Windpocken“ (LKU, 401).

- „Impetigo contagiosa, Grindflechte, Borkenflechte ist eine bakterielle Hautkrankheit, verursacht durch Streptococcus pyogenes oder Staphylococcus aureus “(LKU, 485).

Pendents: $d$. Herpes simplex $\rightarrow n l$. herpes simplex, herpes febrilis (PGW, 506), $d$. Herpes zoster $\rightarrow n l$. herpes zoster, gordelroos, zona (südniederländisch) (PGW, 507), $d$. Impetigo contagiosa $\rightarrow n l$. impetigo contagiosa, impetigo vulgaris (PGW, 559).

Fremde Einworttermini:

- „Malaria (it. malaria ,schlechte Luft') - ist eine Infektion von roten 
Blutkörperchen (Erythrozyten) durch Plasmodien-Parasiten, die durch Stechmücken übertragen werden" (LKU, 614).

- Syphilis (lat. nach dem Titel eines lateinischen Lehrgedichtes des 16. Jhs., in dem die Geschichte eines erkrankten Hirten namens Syphilus erzählt wird).

- „Als Syphilis wird die sexuell übertragbare Infektion mit Treponemia palidum bezeichnet (LKU, 1055).

- „Cholera (gr.); (Syn. Gallenbrechdurchfall;) schwere epidemische Infektionskrankheit mit heftigem Brechdurchfall“ (DUDEN WMF, 199).

- „Als Pertussis (lat. tussis ,Husten') wird die Infektion mit Bordatella pertussis [...] einem gramnegativen Stäbchenbakterium bezeichnet. Syn. Keuchhusten" (LKU, 816).

- „Lepra (gr. ,Aussatz, Krätze, Räude') - Aussatz, chronisch verlaufende, durch Mycobakterium leprae hervorgerufene Infektionskrankheit [...], die zu schweren Verunstaltungen des Körpers, insbesondere der Weichteile oder der Akren, führt“ (DUDEN WMF, 460).

- „Skabies (lat. scabies ,Rauigkeit; Räude, Krätze) - durch die Krätzmilbe hervorgerufene Hautkrankheit mit charakteristischen Milbengängen unter der Oberhaut" (DUDEN WMF, 719).

- „Mumps (engl. pl. mump ,Grimasse'), nach dem angeschwollenem Gesicht, fieberhafte Infektionskrankheit mit schmerzhafter einseitiger Schwellung der Ohrspeicheldrüse, Syn. Ziegenpeter, Parotitis“ (DUDEN DUW, 1042).

- „Tetanus (gr. tetanos,Spannung, lat. tetanus) - Wundstarrkrampf, nach Infektion einer Wunde auftretende Krankheit, die sich durch Muskelkrämpfe, Fieber u.ä. äußert“ (DUDEN DUW, 1527).

Pendents: $d$. Malaria $\rightarrow n l$. malaria (PGW, 680), $d$. Syphilis $\rightarrow n l$. syfilis, syphilis (PGW, 1095), $d$. Cholera $\rightarrow n l$. cholera (PGW, 210), $d$. Pertussis /Keuchhusten $\rightarrow n l$. pertussis, kinkhoest (PGW, 864), $d$. Lepra $\rightarrow n l$. lepra, melaatsheid, ziekte van Hansen, morbus Hansen (PGW, 634), d. Skabies $\rightarrow n l$. scabies, schurft (PGW, 1006), d. Mumps $\rightarrow n l$. bof, parotitis epidemica, epidemische parotitis (PGW, 844), $d$. Tetanus $\rightarrow n l$. tetanus (PGW, 1156).

\section{Akronyme (aus dem Englischen)}

- AIDS (Abk. für engl. Acquired immune deficiency syndrome = erworbenes Immundefektsyndrom" (DUDEN WMF, 90).

- „SARS (Abk. engl. Severe acute respiratory syndrome) „schweres akutes respiratorisches Syndrom - akute Virusinfektion mit dem Erscheinungsbild einer atypischen Pneumonie, hervorgerufen durch Coranaviren" (DUDEN WMF, 705).

Pendents: $d$. AIDS $\rightarrow n l$. aids (PGW, 27), $d$. SARS $\rightarrow n l$. SARS (PGW, 1033)

\section{Morphologische Einteilung der Namen von Infektionskrankheiten im Niederländischen}

Pluralia tantum: mazelen ( $d$. Masern), pokken ( $d$. Pocken), waterpokken $(d$. Windpocken) 
Simplizia: bof (d. Mumps), schurft (d. Skabies), zona (d. Herpes zoster)

Determinativkomposita: gasgangreen ( $d$. Gasbrand), gordelroos (d. Gürtelrose), hondsdolheid (d. Tollwut), kinkhoest (d. Pertussis), knokkelkooorts (d. Denguefieber), kattenkrabziekte (d. Katzenkratzkrankheit), miltvuur (d. Milzbrand), papegaaienziekte (d.Papagaienkrankheit), prionziekte (d. Creutzfeldt-JakobErkrankung), roodvonk (d. Scharlach), slaapziekte ( $d$. Trypanosomiasis), vogelgriep (d. Vogelgrippe), wondroos (d. Wundrose), waterpokken (d. Windpocken).

Determinativkomposita mit Geonymen: rangoonkoorts ( $d$. Denguefieber), marburgvirus (d. Marburg-Vruskrankheit), lassakoorts/lassa-koorts (d. Lassa-Fieber), Lyme-borreliose ( $d$. Borreliose).

Hybride Bildungen: safariziekte (d. Trypanosomiasis)

Suffixbleitungen: -ose: aspergillose (d. Aspergillose), legionellose (d. Legionellose); -itis: encefalitis (d. Enzephalitis); -isme: botulisme (d. Botulismus), -iasis: trypanosomiasis (d. Trypanosomiasis); -ie: pneumonie (d. Pneumonie)

Wortgruppe (Adjektiv/Ordinalzahl + Substantiv): Siberische pest $(d$. Milzbrand), gele koorts ( $d$. Gelbfieber), vijfdaagse koorts ( $d$. Dengue-Fieber), epidemische parotitis ( $d$. Mumps), vijfde ziekte ( $d$. Ringelröteln, Fünfte Krankheit), rodehond (d. Röteln)

Wortgruppe mit einem Eponym: Van der Scheerse koorts (d. Denguefieber), ziekte van Pfeiffer / klierkoorts van Pfeiffer ( $d$. Pfeiffer'sches Drüsenfieber), ziekte van Creutzfeldt-Jakob ( $d$. Creutzfeldt-Jakob-Erkrankung), ziekte van Hansen ( $d$. Morbus Hansen, Lepra)

Wortgruppe mit einem Geonym: hemorrhagische Lassa-koorts (d. Lassa-Fieber)

Entlehnungen: dengue ( $d$. Denguefieber), cholera $(d$. Cholera), malaria $(d$. Malaria), pertussis (d. Pertussis, Keuchhusten), scabies (d. Skabies), syfilis (d. Syphilis)

Griechische und Lateinische Fremdwörter (Wortgruppe): herpes simplex, erythrema infectiosum ( $d$. Ringelröteln), herpes neonatorum, impetigo contagiosa ( $d$. Grindflechte, Borkenflechte), parotitis epidermica

\section{Semantische Einteilung der Krankheitsnamen}

Es gibt verschiedene Motive der Benennung und Klassifikation. Leiber und Olbrich (1981:XXIII) nennen neun Prinzipien, nach denen man Namen für die gebräuchlichen Syndrome (Komplexe von Symptomen) erfunden hat. $\mathrm{Zu}$ diesen Prinzipien gehören: Benennung mit Autoren- oder Patientennamen, mit aus der Volkssprache entstammenden Bezeichnungen, nach der Ätiologie, nach der Pathogenese, nach Hauptsymptomen, nach der anatomischen Symptomlokalisation, nach pathologisch-anatomischen Hauptbefunden und nach dem Anfangsbuchstaben der Hauptsymptome.

Für die Bezeichnungen von den 49 hier dargestellten Infektionskrankheiten lassen sich folgende Kategorien aufstellen. Manchmal kann man einen bestimmten Krankheitsnamen in mehr Kategorien einordnen: 
- Autorenname (Eponym)

d: Pfeiffer'sches Drüsenfieber, Creuzfeldt -Jakob-Erkrankung/ nl. Pfeiffer-ziekte, klierkoorts van Pfeiffer, ziekte van Hansen, ziekte van Creutzfeldt-Jakob, Van der Scheerse koorts

- Ort, wo die Krankheit entdeckt worden ist (Geonym)

$d$. Marburg-Viruskrankheit, $d$. Lassa-Fieber, $d$. Lyme-Borreliose/ nl. marburgvirus, lassa-koorts/lassakoorts; hemorragische Lassa-koorts, rangoonkoorts

- Volkssprache

d. Ziegenpeter, Keuchhusten, Tollwut, Siebentagefieber, Schlafkrankheit/nl. roodvonk, bof, hondsdolheid

- Ätiologie (Krankheitserreger)

Parasiten/Pilze als Krankheitserreger: $d$. / $n l$. Aspergillose, Malaria

Bakterielle Krankheiten: $d$. Scharlach, $d$. Milzbrand, Tuberkulose/ nl. roodvonk, miltvuur, tuberculose,

Viruskrankheiten: d. Marburgviruskrankheit, Pfeiffer'sches Drüsenfieber, Masern, Röteln, Gelbfieber, Denguefieber / nl. marburgvirus, Pfeifferziekte, mazelen, rodehond, gele koorts, dengue

Prione als Krankheitserreger $-d$. Creutzfeldt-Jakob-Erkrankung/ $n l$. ziekte van Creutzfeldt-Jakob

Protozoen als Krankheitserreger: $d$. Toxoplasmose/ $n l$. toxoplasmose/osis

Hauptsymptome: $d$. Röteln, Scharlach/nl. rodehond, roodvonk

Symptomlokalisation: $d$. Pneumonie, $n l$. longontsteking (Lungen), $d$. Enzephalitis, $n l$. encefalitis (Gehirn), $d$. Meningitis, $n l$. meningitis (Hirnhäute)

\section{Abschließende Bemerkungen}

In der Arbeit wurden 49 Infektionskrankheiten dargestellt. Im deutschsprachigen Korpus wurden 66 Namen dieser Krankheiten angetroffen, im niederländischen beläuft sich die Zahl ihrer Pendents auf 86. Im Niederländischen werden häufiger Synonyme für bestimmte Krankheiten verwendet, obwohl auch im Deutschen Synonyme für bestimmte Krankheitsnamen vorkommen. Im ersten Teil der Untersuchung wurden Krankheitsnamen auf ihren Bau untersucht und es wurde festgestellt, dass sie unterschiedliche morphologische Strukturen aufweisen. Darunter gibt es Pluralia tantum, Determinativkomposita, Eponyme und Akronyme, Ableitungen mit und ohne Suffix, auch von fremden Basen, hybride Bildungen, Wortgruppen. In beiden Sprachen gibt es Beispiele von Krankheitsnamen, die auf die gleiche Weise gebildet werden, wie $d$. Vogelgrippe und nl. vogelgriep oder $d$. Katzenkraztkrankheit nl. kattenkrabziekte. In den genannten Krankheitsnamen sind die Pendents 1:1. Es gibt aber auch Belege, wo nur ein Krankheitsname im Deutschen vorkommt, (z.B. Denguefieber) - dem sogar sieben Varianten im Niederländischen entsprechen: dengue, knokkelkoorts, vijfdaagse koorts, dandy fever, forest fever, rangoonkoorts, Van der Scheerse koort.

Nicht alle Pluralia tantum im Deutschen sind auch Pluralia tantum im 
Niederländischen, z.B. d. Röteln (Pl) und nl. rodehond (K) dafür sind d. Masern nl. mazelen in beiden Sprachen Pluralia tantum.

Manche Krankheiten haben volkstümliche Namen im Deutschen, z.B. d. Gürtelrose, Grindflechte, Borkenflechte, Katzenkratzkrankheit, Keuchhusten, Siebentagefieber, Tollwut, Windpocken, Wundrose, Ziegenpeter oder im Niederländischen: bof, gele koorts, knokkelkoorts, gordelroos, melaatsheid, miltvuur, mazelen, rodehond, waterpokken, wondroos.

Es ist nicht immer so, dass bei jeder Krankheit in beiden Sprachen eine volkstümliche Bezeichnung verwendet wird. z.B. Ringelröteln im Deutschen besitzt keine niederländischen Pendents, sondern es werden lateinische Bezeichnungen dieser Krankheit gebraucht.

Es gibt auch Lehnübersetzungen, z.B.d. Scharlach und Fremdwörter, vor allem aus dem Griechischen oder aus dem Lateinischen. Die Krankheitsnamen enthalten auch Eponyme, die in einer Aneinanderreihung oder Wortgruppe im Niederländischen vorkommen: ziekte van Hansen, klierkoorts van Pfeiffer, morbus Hansen oder im Deutschen Bestandteile der Komposita sind: Creutzfeldt-Jakob-Erkrankung.

Im Deutschen ist die Kategorie Wortgruppe (Adjektiv + Substantiv) wesentlich kleiner als im niederländischen Korpus. Vor allem können in die Kategorie Entlehnungen mit nachgestelltem Attribut oder Substantive mit vorangestelltem eponymischem Adjektiv eingeordnet werden. Darunter kann man auch Bezeichnungen antreffen, die mit einem zusammengesetzten Adjektiv verwendet werden: $n l$. viffdaagse koorts oder mit einer Ordinalzahl auftauchen, $d$. Fünfte Krankheit, nl. vijfde ziekte.

Manche Krankheitsnamen kommen mit einem Eponym in Form eines vorangestellten adjektivischen Attributs vor, z.B. d. Pfeiffer'sches Drüsenfieber, nl. Van der Scheerse koorts.

Man kann hier auch Bezeichnungen mit Geonymen aussondern: d. MarburgViruskrankheit, nl. marburvirus, (im Niederländischen ist das Wort ziekte ,Krankheit im Namen nicht enthalten), oder nl. rangoonkoorts

Im Niederländischen gibt es auch mehr englischsprachige Bezeichnungen, z.B dandy fever, forest fever,

Sowohl im Deutschen als auch im Niederländischen sind Akronyme gebräuchlich, z.B. AIDS, SARS. In beiden Fällen handelt es sich um aus dem englischen gebildeten Akronyme, die sich auf die relativ neu erfundenen Erkrankungen beziehen.

Trotz der großen Ähnlichkeit beider Sprachen sind im Bereich der Namen von Infektionskrankheiten auch viele Unterschiede nachweisbar, was vor allem die Anzahl der Bezeichnungen betrifft. Der zweite große Unterschied liegt in der morphologischen Form. Im Niederländischen sind die Wortgruppen (Substantiv + Adjektiv) häufiger als im Deutschen. Im Niederländischen treten häufiger Präpositionalgruppen mit der Präposition VAN auf. Die Namen derselben Krankheit, die die Form eines Determinativkompositums haben, weisen nicht immer die gleichen Wörter als Bestandteile auf, z.B.d. Milzbrand $\leftrightarrow$ nl. miltvuur, d. Windpocken $\leftrightarrow n l$. waterpokken.

Die Suffixderivate sind diejenigen Krankheitsnamen, die einander in beiden 
Sprachen ähneln, obwohl hier auch das Suffix -ismus durch -isme im Niederländischen wiedergegeben wird.

Die Namen der Infektionskrankheiten lassen viele Gemeinsamkeiten in beiden Sprachen erkennen, es gibt doch aber viele Unterschiede, die sich nicht nur auf die volkstümlichen Bezeichnungen, sondern auch auf den Fachwortschatz fremder Herkunft beziehen.

\section{Bibliographie}

Bauer, G. 1996. Übergangsformen zwischen Eigennamen und Gattungsnamen. In Namenforschung/Name Studies/Les noms propres. Internationales Handbuch zur Onomastik/ An international Handbook of Onomastics/Manuel international d'Onomastique, E. Eichler, G. Hilty, H. Löfller, H. Steger, L. Zgusta (Hrsg), Bd.2., 1616-1621. Berlin, New York: Walter de Gruyter

Die Bibel. Altes und Neues Testament. Einheitsübersetzung. Freiburg, Basel, Wien: Herder.

Drosdowski, G. (Hrsg.). 1989. DUDEN Deutsches Universalwörterbuch. Mannheim: Bibliographisches Institut \& FA.Brockhaus.

DUDEN. Wörterbuch medizinischer Fachbegriffe. 2007. Mannheim: Bibliographisches Institut \& F. A. Brockhaus.

Leiber, B. und G. Olbrich.1981. Die klinischen Syndrome. München: Urban \&Schwarzenberg. Lexikon der Krankheiten und Untersuchungen. 2008. Stuttgart: Georg Thieme Verlag. Schulze, P. 2003. Fachsprache der Medizin - Wissen ist Verstehen. Neu-Isenburg: LinguaMed. VAN DALE. Groot Woordenboek der Nederlandse Taal. 1995. Utrecht, Antwerpen: Van Dale Lexicografie.

Van Everdingen, J.J.E. et al. 2006. Pinkhof Geneeskundig Woordenboek. Houten: Bohn Stafleu van Loghum. 